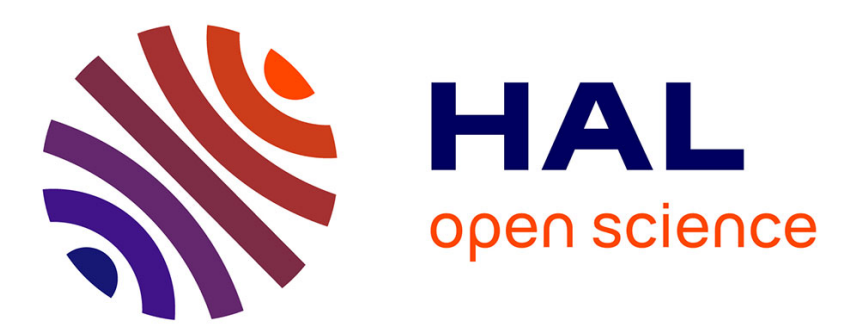

\title{
Antennes imprimées multicouches: choix des fonctions de base dans la méthode des moments
}

\author{
J. Bennegueouche, Jean-Pierre Damiano, A. Papiernik
}

\section{To cite this version:}

J. Bennegueouche, Jean-Pierre Damiano, A. Papiernik. Antennes imprimées multicouches: choix des fonctions de base dans la méthode des moments. Journal de Physique III, 1993, 3 (3), pp.553-562. 10.1051/jp3:1993148 . jpa-00248940

\section{HAL Id: jpa-00248940 https://hal.science/jpa-00248940}

Submitted on 1 Jan 1993

HAL is a multi-disciplinary open access archive for the deposit and dissemination of scientific research documents, whether they are published or not. The documents may come from teaching and research institutions in France or abroad, or from public or private research centers.
L'archive ouverte pluridisciplinaire HAL, est destinée au dépôt et à la diffusion de documents scientifiques de niveau recherche, publiés ou non, émanant des établissements d'enseignement et de recherche français ou étrangers, des laboratoires publics ou privés. 
Classification

Physics Abstracts

$41.90-02.60$

\title{
Antennes imprimées multicouches : choix des fonctions de base dans la méthode des moments
}

\author{
J. Bennegueouche, J. P. Damiano et A. Papiernik \\ Laboratoire d'Electronique, Université de Nice-Sophia Antipolis, CNRS, bât. 4, Les Lucioles 1, \\ 250 rue Albert Einstein, 06560 Valbonne, France
}

(Reçu le 17 mars 1992, révisé le 26 novembre 1992, accepté le 7 décembre 1992)

\begin{abstract}
Résumé. - Nous présentons une méthode théorique pour l'étude des antennes imprimées multicouches à éléments rayonnants de forme circulaire, alimentés par sonde coaxiale. La méthode est basée sur une équation intégrale de réaction, résolue dans le domaine spectral, à l'aide d'une méthode des moments (méthode de Galerkin). L'objectif est d'obtenir les caractéristiques radioélectriques de l'antenne : impédance d'entrée, diagramme de rayonnement, gain, etc. Le choix des fonctions de base est un critère important. Nous avons réalisé une étude théorique de la pondération de ces fonctions sur une large bande de fréquence, d'où des résultats théoriques plus précis, un temps de calcul réduit et un accord théorie-expérience meilleur.
\end{abstract}

\begin{abstract}
We present a theoretical method applied to the analysis of multilayered printed antennas with disc radiating elements, fed by coaxial probe. The method is based on a reaction integral equation solved in the spectral domain using the method of moments (Galerkin's method). The choice of the basis functions is crucial. We study the theoretical ponderation of these functions in a wide frequency band is done to yield more precise numerical results with a reduced computational time. Finally, it shows better agreement with the experimental data.
\end{abstract}

\section{Introduction.}

La méthode théorique exposée concerne les antennes imprimées multicouches à éléments rayonnants en forme de disque, alimentées par sonde coaxiale (Fig. 1). L'étude est basée sur une équation intégrale de réaction, résolue dans le domaine spectral par une méthode des moments (méthode de Galerkin). L'objectif est d'obtenir les caractéristiques radioélectriques de l'antenne : impédance d'entrée, diagrammes de rayonnement, directivité, gain, ... Nous nous intéressons plus particulièrement aux aspects numériques liés au choix des fonctions de base et à leur action sur la précision des résultats.

\section{Analyse.}

L'étude théorique est basée sur une équation intégrale de réaction, résolue dans le domaine spectral à l'aide d'une méthode des moments. Les courants de surface sont décomposés sur une base de fonctions orthogonales dont le choix est un critère important pour la stabilité et la convergence des intégrales mises en jeu [1]. Les fonctions de test sont prises identiques aux 


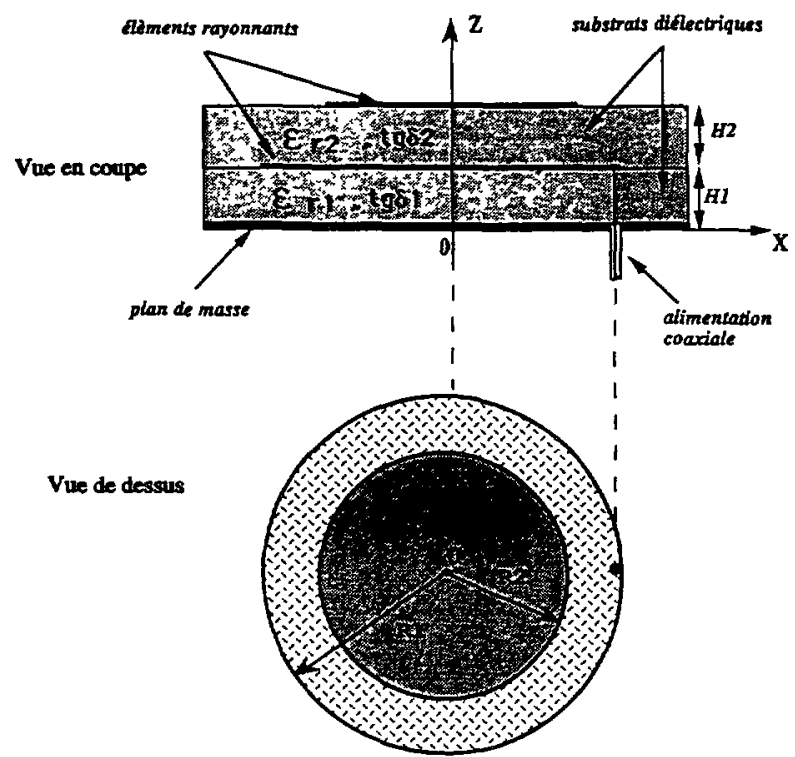

Fig. 1. - Exemple d'une structure d'antenne imprimée multicouche. Les éléments rayonnants sont en forme de disque.

[Multilayer microstrip antenna structure with disc radiating elements (side view and top view).]

fonctions de base (méthode de Galerkin) $[2,3]$, ces fonctions utilisent les modes résonnants du disque, c'est-à-dire les modes TM et TE (dont les expressions sont détaillées en annexe).

2.1 EQUATION DE RÉACTION. - L'équation intégrale de réaction [4] s'écrit pour une antenne imprimée monocouche alimentée par une sonde coaxiale (Fig. 2) :

$$
-\int_{S} \mathbf{J}_{S} \cdot \mathbf{E}_{\mathrm{T}} \mathrm{d} S=\int_{V_{\mathrm{A}}} \mathbf{J}_{\mathrm{A}} \cdot \mathbf{E}_{\mathrm{T}} \mathrm{d} V .
$$

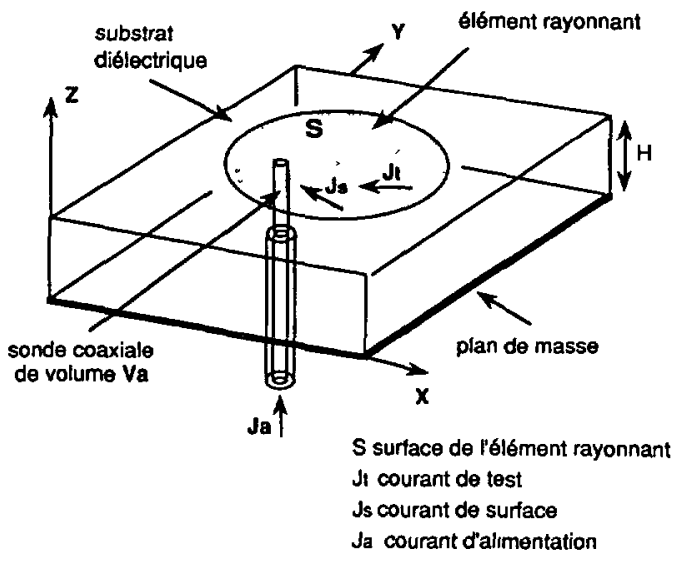

Fig. 2. - Antenne microruban monocouche avec la mention de la surface $S$ de l'élément rayonnant et du volume $V_{\mathrm{A}}$ de la sonde coaxiale.

[Single layer microstrip antenna : $S$ is the area of the radiating element. $V_{\mathrm{A}}$ is the volume of the coaxial feed.] 
Dans le cas de 2 éléments rayonnants de surface $S_{1}$ et $S_{2}$ et $K$ alimentations, nous aurons :

$$
-\int_{S_{1}} \mathbf{J}_{S_{1}} \cdot \mathbf{E}_{\mathrm{T}} \mathrm{d} S-\int_{S_{2}} \mathbf{J}_{S_{2}} \cdot \mathbf{E}_{\mathrm{T}} \mathrm{d} S=\sum_{k=1}^{K} \int_{V_{\mathrm{A}_{k}}} \mathbf{J}_{\mathrm{A}_{k}} \cdot \mathbf{E}_{\mathrm{T}} \mathrm{d} V
$$

où :

$\mathbf{J}_{\mathrm{A}_{k}}$ est la densité volumique du courant d'alimentation (accès $k$ ) dans un volume $V_{\mathrm{A}^{*}}$.

Le courant d'alimentation est modélisé par un courant supposé uniformément réparti sur un cylindre de même diamètre $d_{0}$ que l'âme centrale de la sonde coaxiale et orienté selon OZ, soit :

$$
\mathbf{J}_{\mathrm{A}}=\frac{1}{\pi \cdot d_{0}} \mathbf{Z}
$$

$\mathbf{J}_{S_{1}}, \mathbf{J}_{S_{2}}$ sont les densités de courant surfacique des éléments rayonnants 1 et 2.

$\mathbf{E}_{\mathrm{T}}$ est le champ électrique de test créé par un courant électrique de test pris sur chacun des 2 éléments.

2.2 Relations Champ Électrique - courants DE SURFACE. - Le champ électrique tangentiel est calculé à partir des fonctions dyadiques de Green $[2,5]$ et des courants de surface $\mathbf{J}_{S_{1}}$ et $\mathbf{J}_{S_{2}}$ [4]. La relation s'écrit dans le domaine spectral :

$$
\tilde{\mathbf{E}}=\left[\begin{array}{ll}
{\left[G^{11}\right]} & {\left[G^{12}\right]} \\
{\left[G^{21}\right]} & {\left[G^{22}\right]}
\end{array}\right]\left[\begin{array}{c}
\tilde{\mathbf{J}}_{S_{1}} \\
\tilde{\mathbf{J}}_{S_{2}}
\end{array}\right]=\left[\begin{array}{c}
\tilde{\mathbf{E}}_{1} \\
\tilde{\mathbf{E}}_{2}
\end{array}\right]
$$

où représente la transformée de Fourier.

$G^{i J}$ représente le champ électrique $\mathbf{E}$ localisé sur le conducteur $j$, créé par un courant électrique unitaire pris sur le conducteur $i$.

$$
\left[G^{i j}\right]=\left[\begin{array}{ll}
g_{x x}^{i j} & g_{x y}^{i j} \\
g_{y x}^{i j} & g_{y y}^{l j}
\end{array}\right]
$$

2.3 MÉThOde DES MOMENTS. - La méthode des moments (méthode de Galerkin) consiste à décomposer les courants de surface inconnus $\mathbf{J}_{S_{1}}, \mathbf{J}_{S_{2}}$ sur une base de fonctions orthogonales connues $\mathbf{F}_{1 n}$ et $\mathbf{F}_{2 n}[2]$ :

$$
\mathbf{J}_{S_{1}}=\sum_{n=1}^{N_{1}} I_{1 n} \quad \mathbf{F}_{1 n} \quad \mathbf{J}_{S_{2}}=\sum_{n=1}^{N_{2}} I_{2 n} \quad \mathbf{F}_{2 n}
$$

$N_{1}$ et $N_{2}$ représentent le nombre total de fonctions de base sur chacun des 2 éléments rayonnants. Nous obtenons finalement un système matriciel à $\left(N_{1}+N_{2}\right)$ inconnues :

$$
Z . I=V
$$

$I$ est la matrice des coefficients à déterminer. 
Les éléments des matrices $Z$ et $V$ sont de la forme :

$$
\begin{aligned}
& Z_{m n}^{p q}=-\lambda_{0}^{2} \int_{-\infty}^{+\infty} \int_{-\infty}^{+\infty} \tilde{\mathbf{E}}_{m}^{T_{p}}(\boldsymbol{\alpha}, \boldsymbol{\beta}) \cdot \tilde{\mathbf{F}}_{q n}(-\alpha,-\beta) \mathrm{d} \alpha \mathrm{d} \beta \\
& V_{m}^{p}=\lambda_{0}^{2} \int_{-\infty}^{+\infty} \int_{-\infty}^{+\infty}\left\{\int_{0}^{\mathrm{H}_{1}} \tilde{G}^{q p} \cdot \tilde{\mathbf{F}}_{p m} \mathrm{~d} z\right\} \cdot \tilde{\mathbf{J}}_{A}(-\alpha,-\beta) \mathrm{d} \alpha \mathrm{d} \beta
\end{aligned}
$$

dans le cas d'une alimentation connectée à l'élément inférieur.

Les transformées de Fourier des fonctions de base $F$ sont données en annexe.

2.4 L'INTÉGRATION NUMÉRIQUE. - La difficulté numérique est rencontrée dans les calculs des éléments matriciels $Z_{m n}$ et $V_{m}$. Les fonctions de base sont très oscillantes. Les fonctions de Green présentent des pôles complexes. Une étude précise de tous ces problèmes a été réalisée permettant de bien comprendre comment évaluer les intégrants [1].

Afin de ne considérer qu'un seul intervalle infini d'intégration, nous avons effectué le changement de variables suivant :

$$
\begin{aligned}
& \alpha=\rho \cos \phi \\
& \beta=\rho \sin \phi
\end{aligned}
$$

tel que $\rho$ soit inclus dans $[0,+\infty[$ et $\phi$ inclus dans $[0,2 \pi]$. Par des considérations trigonométriques et pour des raisons de parité des intégrants, il est toujours possible de ramener l'intervalle d'intégration de $\phi$ à $[0, \pi / 2]$.

Afin de bien prendre en compte toutes ces particularités, nous avons découpé le domaine d'intégration de $\rho$ en 4 intervalles du domaine d'intégration selon $\rho$ :

$$
\begin{aligned}
& {[0-1]} \\
& {\left[1-\sqrt{\varepsilon_{\mathrm{r} \max }}\right]} \\
& {\left[\sqrt{\varepsilon_{\mathrm{r} \max }}-\rho_{\max }\right]} \\
& {\left[\rho_{\max }-\infty[\right.}
\end{aligned}
$$$$
\text { méthode classique d'intégration (Gauss) }
$$$$
\text { présence d'au moins un pôle : méthode de contour analytique }
$$$$
\text { d'intégration pour éviter les pôles, développée par Pozar [6] }
$$$$
\text { oscillations : méthode de Gauss }
$$$$
\text { fortes oscillations : méthode des moyennes pondérées [7] }
$$$$
\varepsilon_{\mathrm{r} \max }=\sqrt{\max \left(\varepsilon_{\mathrm{r} 1} \cdot \varepsilon_{\mathrm{r} 2}\right)} ; \quad \rho_{\max }=100 .
$$

Cette valeur 100 est déterminée empiriquement par une étude de la convergence des intégrales mises en jeu.

2.5 L'IMPÉDANCE D'ENTRÉE. - Connaisant les courants de surface sur les éléments rayonnants, nous pouvons déterminer l'impédance d'entrée $Z_{\mathrm{e}}$ comme étant la réaction du courant d'alimentation $J_{\mathrm{A}}$ avec les courants existant sur les éléments rayonnants [8]. Dans le cas d'un courant source d'intensité $I_{0}$, l'impédance d'entrée $Z_{\mathrm{e}}$ de l'antenne s'écrit :

$$
Z_{\mathrm{e}}=-\left(\frac{1}{I_{0}^{2}}\right) \sum_{n=1}^{N} I_{n} \cdot V_{n} \quad \text { avec } \quad N=N_{1}+N_{2}
$$

\section{Etude de la pondération des fonctions de base.}

Les fonctions de base étudiées sont des modes de type $\mathrm{TM}_{n s}$ et $\mathrm{TE}_{m p}$. Dans notre modélisation, nous avons considéré un grand nombre de fonctions, c'est ainsi que nous avons pu déterminer 
de manière précise leur pondération dans la bande de fréquence [1,8-9,2] GHz représentées dans la figure 3 sur une antenne simple monocouche alimentée par une sonde coaxiale. Nous remarquons que tous les modes $\mathrm{TM}_{n s}$ sont excités à la même fréquence pour $n$ pair ainsi que pour $n$ impair, l'excitation est identique pour les modes $\mathrm{TE}_{m p}$. Un pic détermine le mode prépondérant, il peut être considéré alors seul pour le calcul de l'impédance d'entrée ; cependant les autres modes sont nécessaires pour obtenir la polarisation croisée. Par exemple à $F=2 \mathrm{GH} z$, les modes fondamentaux $\mathrm{TM}_{11}$ et $\mathrm{TE}_{11}$ sont dominants, et à $F=4,1 \mathrm{GHz}$ c'est au tour des modes $\mathrm{TM}_{21}$ et $\mathrm{TE}_{21}$ d'être prépondérants.

La figure 4 montre les résultats théoriques et expérimentaux de l'impédance d'entrée dans la bande $[3,9-4,5] \mathrm{GHz}$ pour une antenne imprimée bicouche à deux éléments rayonnants ;
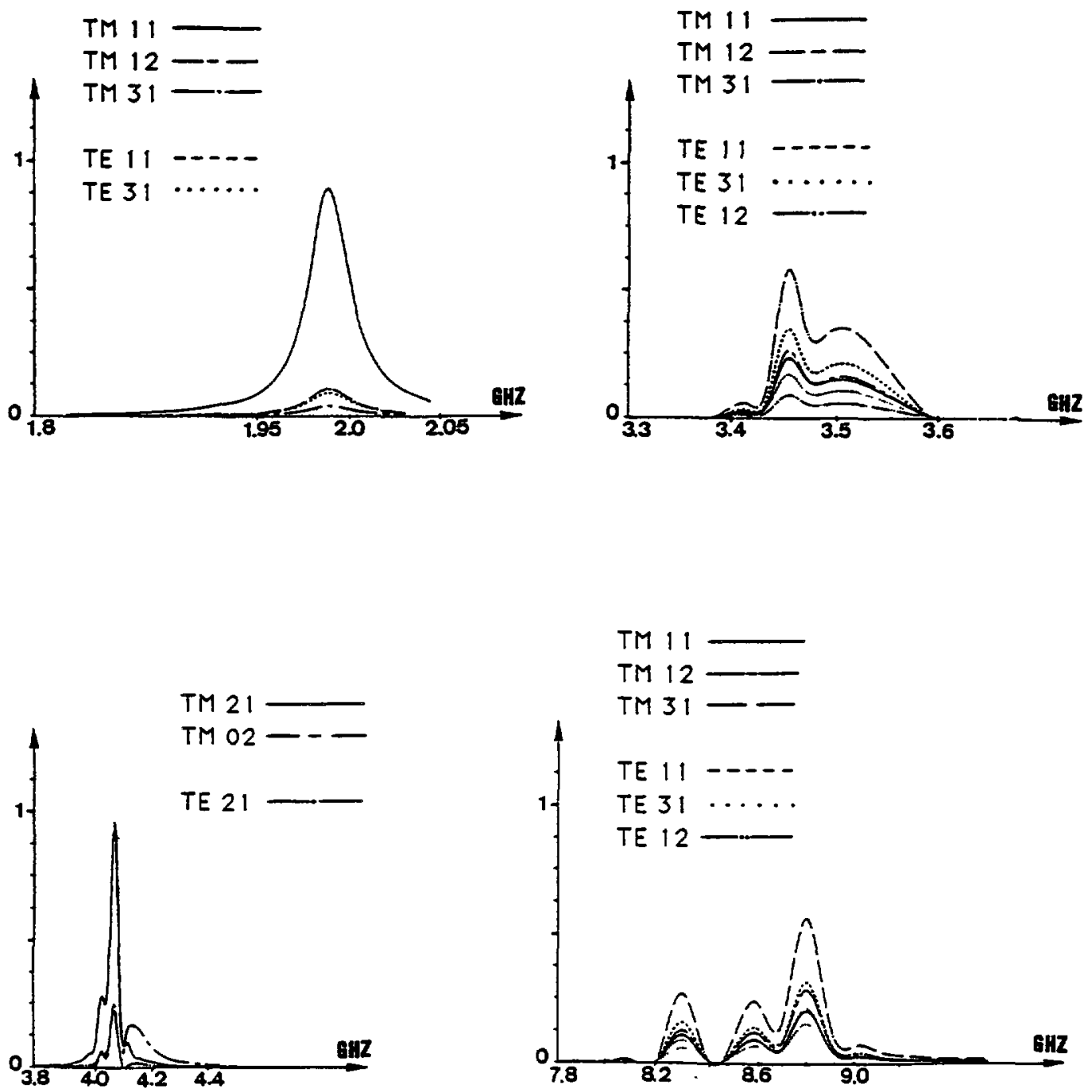

Fig. 3. - Variations des coefficients de pondération des fonctions de base de type TM et TE. Avec $R=2,865 \mathrm{~cm}, \varepsilon_{\mathrm{r}}=2,2 ; \operatorname{tg} \delta=0,001 ; H_{1}=0,1524 \mathrm{~cm}, X_{\mathrm{a}}=0,7 \mathrm{~cm} ; d_{0}=0,065 \mathrm{~cm}$.

[Study of the weighting coefficients of the basis functions versus the frequency.] 


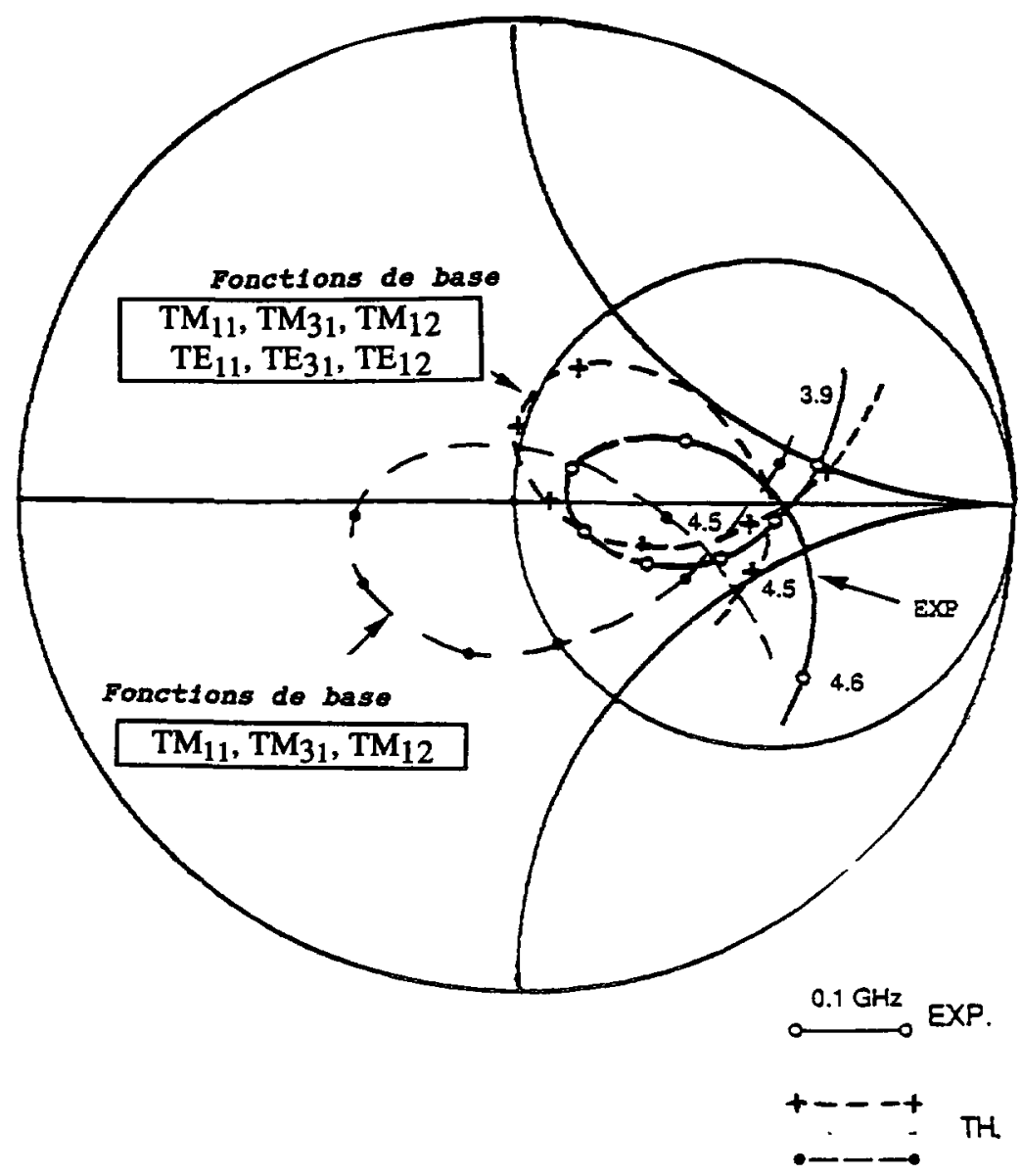

Fig. 4. - Lieux d'impédance d'entrée d'une antenne bicouche à deux éléments rayonnants en forme de disque. Mise en évidence de la nécessité de bien sélectionner les fonctions de base. $R_{1}=1,22 \mathrm{~cm}$, $R_{2}=1,21 \mathrm{~cm}, \varepsilon_{r_{1}}=2,33, \operatorname{tg} \delta_{1}=0,0012, H_{1}=3,18 \mathrm{~mm}, \varepsilon_{r_{2}}=2,33, \operatorname{tg} \delta_{2}=0,0012, H_{2}=1,59 \mathrm{~mm}$, $d_{0}=0,65 \mathrm{~mm}, X_{\mathrm{a}}=11,8 \mathrm{~mm}, Z_{0}=50$ ohms.

[Input impedance of a multilayer microstrip antenna with two disc radiating elements. Influence of the choice of the basis functions.]

l'alimentation étant connectée à l'élément inférieur. Dans nos calculs, nous avons pris en compte dans un premier temps les modes théoriques $T M_{11}, T_{31}$ et $T M_{12}$, puis nous avons rajouté les mêmes modes théoriques de type TE sur les conducteurs. Ceci entraîne une nette amélioration des résultats théoriques. L'accord théorie-expérience est satisfaisant.

Les calculs ont été effectués sur un VAX 5500. Le programme de simulation est écrit en Fortran 77. Par exemple, le temps de calcul d'une structure bicouche à deux éléments rayonnants est de l'ordre de $3 \mathrm{mn}$ pour deux fonctions de base sur chacun des disques et de $8 \mathrm{mn}$ pour quatre fonctions de base.

\section{Conclusion.}

L'originalité de cette étude numérique consiste en un choix judicieux des fonctions de base théoriques utilisées lors des calculs des caractéristiques radioélectriques d'une antenne 
imprimée multicouche à éléments rayonnants en forme de disque. Cela nous a permis d'obtenir, pour un temps de calcul réduit, des résultats théoriques en bon accord avec l'expérience.

\section{Annexe.}

\section{Expressions des fonctions de base.}

Nous donnons les expressions des fonctions de base de type $\mathrm{TM}_{n s}$ et $\mathrm{TE}_{m p}$ en coordonnées polaires et dans le domaine spectral. Nous donnons en outre quelques représentations graphiques de ces fonctions à savoir par exemple, celles de type $\mathrm{TM}_{11}, \mathrm{TM}_{21}, \mathrm{TE}_{11}$ et $\mathrm{TE}_{21}$ (Figs. 5 et 6 ).

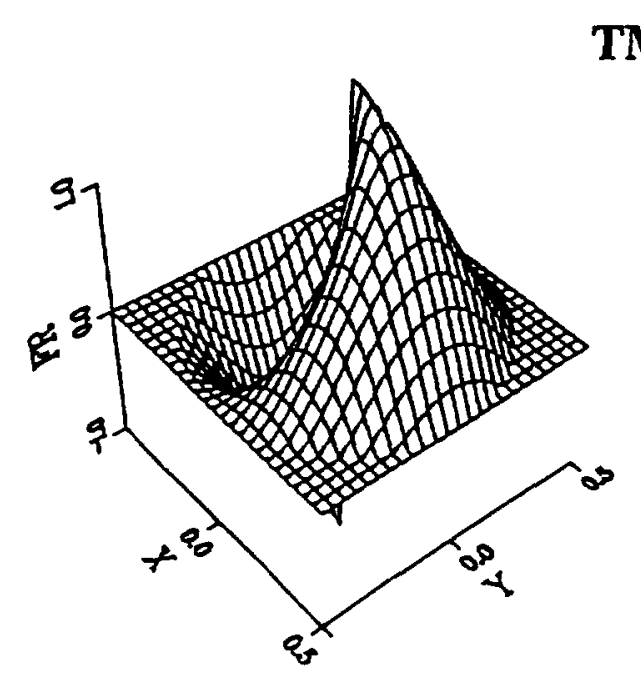

\section{TM 11}

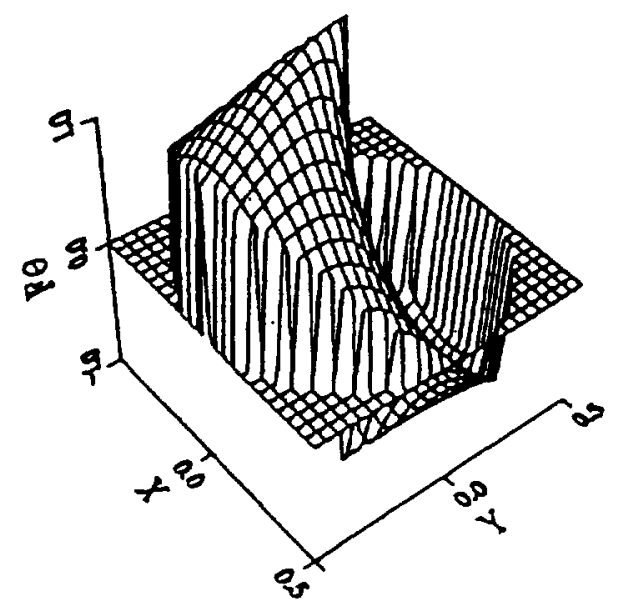

\section{TM 21}
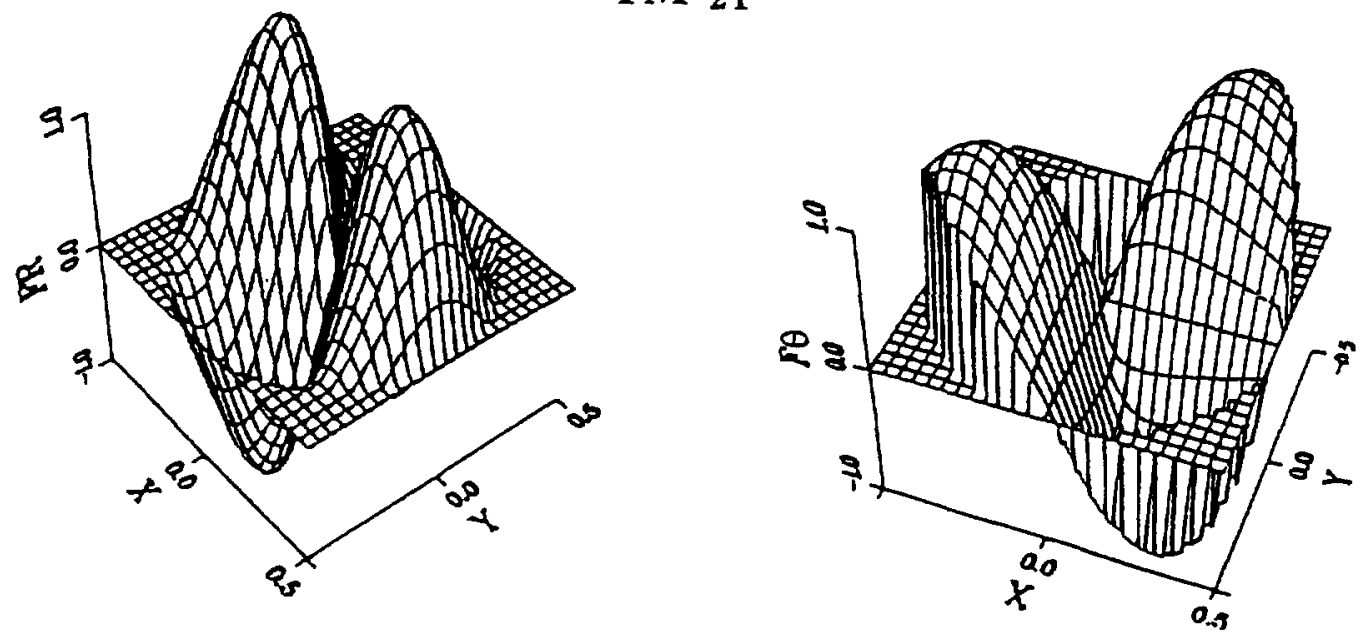

Fig. 5. - Variations des composantes $F_{r}$ et $F_{\theta}$ des fonctions de base $\mathrm{TM}_{n s}$.

[Graphic representation of the radial and azimuthal components of the $\mathrm{TM}_{11}$ and $\mathrm{TM}_{21}$ basis functions.] 


\section{TE 11}
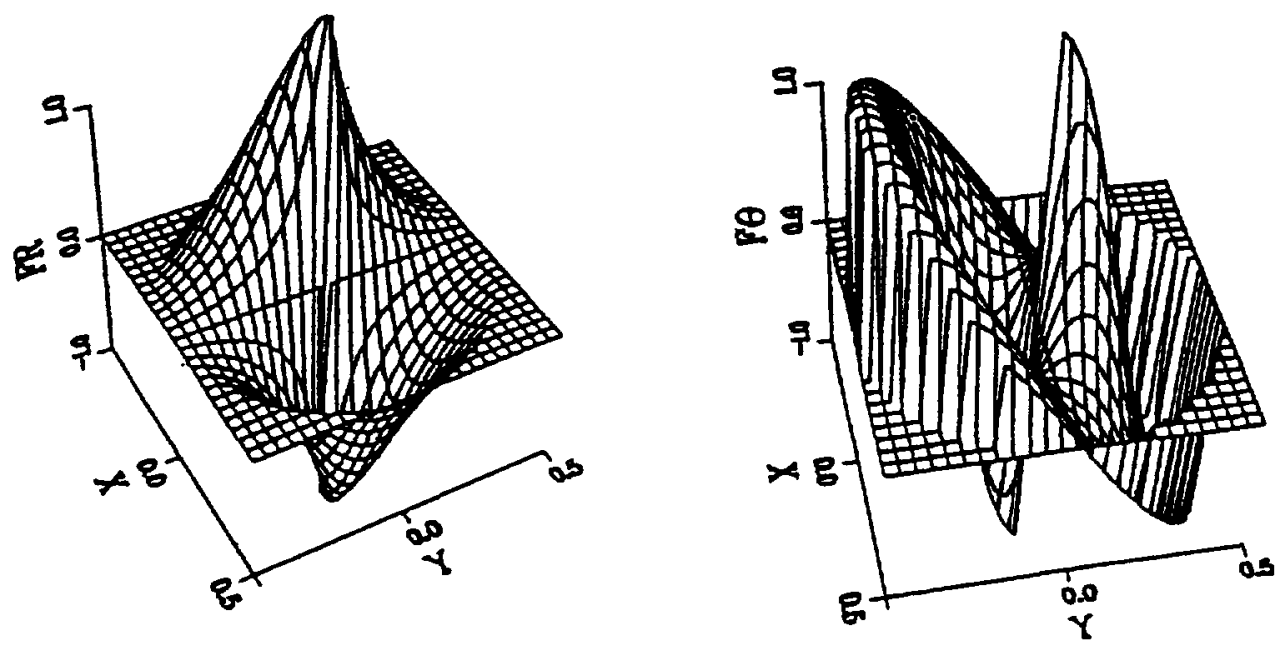

TE 21
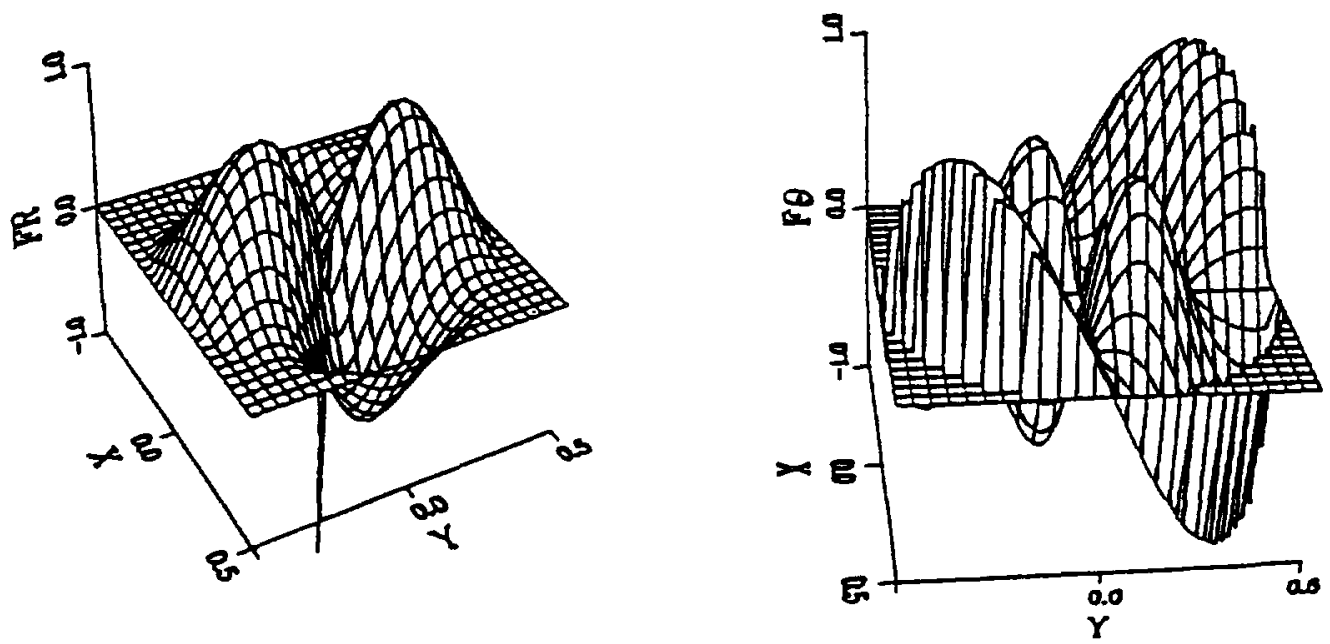

Fig. 6. - Variations des composantes $F_{r}$ et $F_{\theta}$ des fonctions de base $\mathrm{TE}_{m p}$.

[Graphic representation of the radial and azimuthal components of the $\mathrm{TE}_{11}$ and $\mathrm{TE}_{21}$ basis functions.] 
FONCTION DE BASE DE TYPE TM Ts

$$
\begin{aligned}
& F_{r}^{n s}(r, \theta)=C \cdot\left(\frac{x_{n s}^{\prime}}{R}\right) \cdot J_{n}^{\prime}\left(\frac{x_{n s}^{\prime} \cdot r}{R}\right) \cdot \cos n \theta \\
& F_{\theta}^{n s}(r, \theta)=-C \cdot\left(\frac{n}{r}\right) \cdot J_{n}\left(\frac{x_{n s}^{\prime} \cdot r}{R}\right) \cdot \sin n \theta
\end{aligned}
$$

avec

$$
C=\frac{\left(\frac{x_{n s}^{\prime}}{R}\right)\left[\frac{\varepsilon_{n}}{\Pi \cdot\left(x_{n s}^{\prime 2}-n^{2}\right)}\right]^{\frac{1}{2}}}{J_{n}\left(x_{n s}^{\prime}\right)}
$$

$R$; rayon du disque, $J_{n}(x)$; la fonction de Bessel de première espèce d'ordre $n, J_{n}^{\prime}(x)$; la dérivée de la fonction de Bessel d'ordre $n, x_{\mathrm{ns}}^{\prime}$; la racine d'ordre $s$ de la dérivée de Bessel d'ordre $n: J_{n}^{\prime}\left(x_{\mathrm{ns}}^{\prime}\right)=0$

$$
\varepsilon_{n}=\left\{\begin{array}{lll}
2 & \text { si } & n=0 \\
1 & \text { si } & n \neq 0
\end{array}\right\}
$$

FONCTION DE BASE DE TYPE TE $m p$

$$
\begin{gathered}
F_{r}^{m p}(r, \theta)=-D \cdot\left(\frac{m}{r}\right) \cdot J_{m}\left(\frac{x_{m p} \cdot r}{R}\right) \cdot \cos m \theta \\
F_{\theta}^{m p}(r, \theta)=D \cdot\left(\frac{x_{m p}}{R}\right) \cdot J_{m}^{\prime}\left(\frac{x_{m p} \cdot r}{R}\right) \cdot \sin m \theta \\
D=\frac{\sqrt{\frac{\varepsilon_{m}}{\pi}}}{R \cdot J_{m+1}\left(x_{m p}\right)}
\end{gathered}
$$

où $x_{m p}$ est la racine d'ordre $p$ de la fonction de Bessel d'ordre $m$ :

$$
J_{m}\left(x_{m p}\right)=0 .
$$

Les fonctions de base calculées dans le domaine spectral sont exprimées selon $\mathrm{O} x$ et Oy par :

FONCTION DE BASE DE TYPE TM $_{n s}$

$$
\begin{gathered}
\tilde{F}_{x}^{n s}(\rho, \phi)=C_{n s}\left\{\begin{array}{c}
x_{n s}^{\prime 2}\left[J_{n-1}\left(k_{0} \rho R\right) \cos (n-1) \phi-J_{n+1}\left(k_{0} \rho R\right) \cos (n+1) \phi\right] \\
+n k_{0} \rho R J_{n}\left(k_{0} \rho R\right)[\cos (n+1) \phi-\cos (n-1) \phi]
\end{array}\right\} \\
\tilde{F}_{y}^{n s}(\rho, \phi)=C_{n s}\left\{\begin{array}{c}
x_{n s}^{\prime 2}\left[-J_{n+1}\left(k_{0} \rho R\right) \sin (n+1) \phi-J_{n-1}\left(k_{0} \rho R\right) \sin (n-1) \phi\right] \\
+n k_{0} \rho R J_{n}\left(k_{0} \rho R\right)[\sin (n+1) \phi+\sin (n-1) \phi]
\end{array}\right\} \\
C_{n s}=j^{n-1} \frac{x_{n s}^{\prime} \sqrt{\frac{\varepsilon_{n} \cdot \Pi}{x_{n s}^{\prime 2}-n^{2}}}}{\lambda_{0}^{2}\left(x_{n s}^{\prime 2}-k_{0}^{2} \rho^{2} R^{2}\right)} \\
\varepsilon_{n}=\left\{\begin{array}{ll}
2 & \text { si } n=0 \\
1 & \text { si } n \neq 0
\end{array}\right\} . \\
J_{n}(x) \text { Bessel function; } J_{n}^{\prime}\left(x_{n s}^{\prime}\right)=0 .
\end{gathered}
$$


FONCTION DE BASE DE TYPE TE $_{m p}$

$$
\begin{aligned}
& \tilde{F}_{x}^{m p}(\rho, \phi)=D_{m p} \cdot k_{0} \rho r J_{m}\left(k_{0} \rho r\right) \cdot[\cos (m+1) \phi-\cos (m-1) \phi] \\
& \tilde{F}_{y}^{m p}(\rho, \phi)=D_{m p} \cdot k_{0} \rho r J_{m}\left(k_{0} \rho r\right) \cdot[\sin (m+1) \phi+\sin (m-1) \phi] \\
& D_{m p}=j^{m-1} \frac{x_{m p} \sqrt{\Pi}}{\lambda_{0}^{2}\left(x_{m p}^{2}-k_{0}^{2} \rho^{2} r^{2}\right)} \\
& J_{m}\left(x_{m p}\right)=0 .
\end{aligned}
$$

\section{Bibliographie}

[1] Damiano J. P., Computation of input impedance in microstrip antennas. Graphic representation and numerical integration of oscillating functions, IEE Proceedings, U.K., 1987, Microwaves Antennas Propag. 134 (1987) 456-466.

[2] Damiano J. P., Bennegueouche J., Papiernik A., Study of multilayer microstrip antennas with radiating elements of various geometry, IEE Proceedings, U.K., 1990, Microwaves Antennas Propag. 137 (1990) 163-170.

[3] Damiano J. P., Bennegueouche J., Papiernik A., Antennes microrubans multidiélectriques : analyse de structures à géométrie mixte : rectangle-disque, Journées Internationales de Nice sur les Antennes (J.I.N.A.), Nice, F., (8-10 nov. 1988) pp. 225-228.

[4] RumSEY V. H., Reaction concept in electromagnetic theory, Phys. Rev. U.S.A. 94 (1954) 1483-1491.

[5] TAI C. T., Dyadic Green's functions in Electromagnetic theory, Scraton PA (Intext Educational Publishers, Collège division of Intext, London 1971).

[6] PozAR D., Input impedance and mutual coupling of rectangular microstrip antennas, I.E.E.E. Trans. A.P., U.S.A. 30 (1982) 68-76.

[7] Mosig J. R., Les structures microrubans : analyse au moyen des équations intégrales, Doctoral thesis, Lausanne, Suisse (1983) Ecole polytechnique Fédérale de Lausanne.

[8] Harrington R. F., Time harmonic electromagnetic fields (McGraw-Hill, New York, 1961). 